\title{
The Roles of Environmental Pollutants in the Pathogenesis and Prevalence of Diabetes: A Review
}

\author{
${ }^{* 1}$ YAHAYA, T; ${ }^{2}$ OBAROH, I; ${ }^{3}$ OLADELE, EO \\ 1. Department of Biology, Federal University Birnin Kebbi, Kebbi State \\ ${ }^{2}$. Department of Biological Sciences, Kebbi State \\ University of Science and Technology, Aliero, Kebbi State \\ ${ }^{3}$. Biology Unit, Distance Learning Institute, University of Lagos. \\ *Correspondence: yahayatajudeen@gmail.com \\ $+234-8033550788,+234-8098233774$
}

\begin{abstract}
Diabetes is on the rise worldwide with a growing suspicion of association between environmental pollutants and diabetes. This paper reviewed the roles of environmental pollutants in the pathogenesis and increasing incidence of diabetes. Relevant information was retrieved from reliable sources in the internet using Google search engine. The review found that studies have established environmental pollutants aid in the pathogenesis of diabetes by causing blood vessel rupture and insulin resistance. Toxic chemicals in pollutants may destroy or cause mutation in pancreatic $\beta$-cells, disrupting its insulin production. People are therefore advised to live far away from polluted or industrial environment. () JASEM

http://dx.doi.org/10.4314/jasem.v21i1.1
\end{abstract}

Keywords: Diabetes, Pathogenesis, Pancreas, Mutation, Insulin, Blood vessel.

\section{INTRODUCTION}

Diabetes is a chronic disease that occurs either when the pancreas does not produce enough insulin or when the body cannot effectively use the insulin it produces. Insulin is a hormone produced by beta cells in the islets of Langerhans in the pancreas and regulates blood glucose (USNIH, 2014). There are three main types of diabetes, namely type 1 , type 2 , and gestational diabetes. Type 1 diabetes is an autoimmune disease which occurs when the body produces too little or no insulin and glucose accumulate in the blood. It develops most often in young people, however, it can also develop in adults. Type 2 diabetes can affect people of all ages, even children. However, it most often develops in middleaged and older people when the pancreas suddenly stops producing insulin, or the body does not respond to the insulin produced. Gestational diabetes is a temporary condition that occurs during pregnancy, but it poses an increased risk of developing diabetes for both mother and child. A common effect of uncontrolled diabetes is a raised blood sugar (hyperglycemia) which may over time cause serious damage to the body, especially the nerves and blood vessels. Other effects of diabetes include increased risk of heart attacks and strokes, foot ulcers, infection and eventual need for limb amputation, blindness, and kidney failure (USNIH, 2014).

Diabetes is on the rise in many countries with the attendant huge burden on national development. About 415 million people worldwide were diagnosed with diabetes in 2015, of which about 4 million cases were recorded in Nigeria (IDF, 2015). More worrisome than the high incidence of diabetes is its high mortality. In 2012, diabetes was the direct cause of 1.5 million deaths and high blood glucose was the cause of another 2.2 million deaths, worldwide (ADA, 2015). In Nigeria, 105, 091 deaths were recorded from diabetes in 2012 (IDF, 2013), and 40,815 adults alone died from the disease in 2015 . Aside the burden of health, diabetes has caused a huge economic burden too. Worldwide, a huge sum of 174 billion dollars was spent on diagnosed diabetes in 2007, and in 2012, the total cost was 245 billion dollars (ADA, 2015). In Nigeria, the cost per person with diabetes was 212.3 dollars in 2015. Over $99 \%$ of all the reported cases of diabetes are type 2 diabetes mellitus mostly in low-to-middle income countries (ADA, 2015).

The increasing incidence of diabetes is worrisome and has been generally attributed to increasing weight gain (obesity), poor diets, and lack of exercise. However, in the last few decades, environmental chemicals have also been suspected in the pathogenesis of diabetes (Lee et al., 2006). Many surveys and some scientific studies have shown a high incidence of diabetes among people living in polluted environment, but it is still not clear the mechanisms by which environmental pollutants cause diabetes. Therefore, this paper was intended to review and provide evidences linking pollutants to the increasing incidence of diabetes. 


\section{MATERIALS AND METHOD}

Google search engine was used to search for current information on diabetes types, causes and prevalence. The search was first done for global information on diabetes and later narrowed down to Nigeria. Literature were sourced from reliable sources such as PubMed, Google Scholar, Medline, Agencies, Blogs, among others.

\section{RESULTS AND DISCUSSION}

Although association between environmental pollutants and diabetes is a neglected issue, all the articles reviewed have established a link between pollutants and diabetes. The link was first observed by Lockwood in 2002 in the United States of America. Lockwood found that states with high toxic emissions had high prevalence of diabetes compared with states with low emissions (Lockwood, 2002). However, he could not explain the nature of association between them. Since then many researchers have tried to explain the association between pollutants and diabetes using human and animal studies.

Evidence from Human Studies: A study from Chile found elevated levels of particulate matter in children that developed type 1 diabetes, suggesting pollutants may play a role in the pathogenesis of diabetes (González et al., 2013). A German study also found that high exposure to traffic-related particulate matter accelerates the pathogenesis of type 1 diabetes, but only in very young children (Beyerlein et al,. 2015). A number of long-term studies have found that exposure to traffic-related air pollution is associated with an increased risk of type 2 diabetes in adults. Adults in Denmark had an increased risk of diabetes when exposed to higher levels of traffic-related air pollutant containing nitrogen dioxide (Andersen et al., 2012). A study conducted among nurses also in Denmark found that those who lived in areas with higher levels of traffic-related pollutants had a higher risk of developing type 2 diabetes. The associations were strongest in non-smokers, obese people, and heart disease patients (Hansen et al., 2016). Although toxicity differs among traffic-related pollutants, they can cause oxidation of lipids and proteins, or activate intracellular oxidant pathways. So, production of oxidative stress is a possible mechanism by which traffic-related pollutants cause insulin resistance. In addition, some studies have reported that short-term and long-term increases in particulate matter and $\mathrm{NO}_{2}$ exposure may lead to elevated inflammatory biomarkers (Lodovici and Bigagli, 2011).

In 2006, a South Korean team found that people with diabetes tended to have elevated concentrations of pollutants including dioxins in their blood. They also discovered that all the risks of diabetes attributed to obesity are actually caused by persistent organic pollutants, and that obesity is only a vehicle for such chemicals (Portal, 2006). Interestingly, an earlier study had shown a high prevalence of diabetes among US veterans who were exposed to dioxins during the Vietnam War (Kern et al., 2004). A Taiwanese study found that people living near contaminated sites with higher levels of dioxin had higher levels of insulin resistance (Chang et al., 2010). A follow-up study by the same authors found that men with the highest dioxin levels had insulin resistance 5-fold higher than those with the lowest levels (Chang et al., 2016). Dioxins are formed during combustion of plastics, particularly in municipal and medical waste incinerators, and may alter insulin metabolism. Dioxins can concentrate in body fats, thus obese individuals are likely to have an increased dioxin body burden.

In a prospective follow-up study, the incidence of type 2 diabetes was two- to fivefold higher in residents chronically exposed to arsenic compared with subjects living in low-exposure areas. In the study, type 2 diabetes was much more frequent in older age groups who experienced long-term arsenic exposure. In America, a survey of 788 adults in 20032004 showed that participants with type 2 diabetes had a $26 \%$ higher level of arsenic in their urine than those without the disease (Wilbert, 2008), There are several possible mechanisms by which inorganic arsenic may contribute to diabetes. Insulin-sensitive cells that are exposed to insulin and arsenic appear to take in less glucose than cells exposed only to insulin. Arsenic could influence genetic factors that interfere with insulin sensitivity and other processes. Arsenic may also contribute to oxygen-related cell damage, inflammation, and cell death, all of which are linked to diabetes (Wilbert, 2008).

Several cross-sectional studies showed an association between Bisphenol A (BPA) and phthalate and type 2 diabetes. In 2012, United States Pediatric Environmental Health Specialist Units (USPEHSU) reported that exposure to BPA during pregnancy could cause obesity and diabetes (Waxman, 2016). A study from the Miguel Hernandez University in Spain reported that BPA can trigger the release of almost double the insulin actually needed to break down food. High insulin levels can desensitize the body to the hormone over time, which in some people may then lead to weight gain and type 2 diabetes (Lynne, 2012). In a 19-year follow-up human study, the incidence of type 2 diabetes increased with the plasma levels of hexachlorobenzene. In the same 
study, the incidence of type 2 diabetes in subjects with a high total polychlorinated biphenyl plasma concentration was 1.7-fold higher than that of subjects with a low concentration (Jean et al., 2015). A prospective cohort study also reported urinary BPA and butyl phthalate were associated with type 2 diabetes (Jean et al, 2015). BPA and phthalate are chemicals used in making plastic products. These chemicals can leach from plastic containers into foods and beverages, disrupting glucose homeostasis, insulin secretion and adipogenesis (Jean et al., 2015).

Evidence from Animal Studies: A recent study showed that rats fed a crude fish oil diet containing a low-dose POPs mixture developed visceral obesity, hepatosteatosis and insulin resistance. POPs in the fish oil elevate total cholesterol levels and aggravate insulin resistance. POPs have also been linked with metabolic syndrome, which predisposes to type 2 diabetes and shares the underlying pathogenesis of insulin resistance with type 2 diabetes (Jean et al., 2015).

Researchers from Duke University exposed a group of lab rats to Beijing air and another group to filtered air for 19 days. At the end of exposure, the group that got the highly polluted Beijing air had higher bad cholesterol levels (50\%), triglycerides (46\%) and total cholesterol $(97 \%)$. These are factors that can increase the risk of developing obesity and diabetes. The male rats in the group exposed to Beijing air were $18 \%$ heavier than their filtered air counterparts, while the female rats were $10 \%$ heavier. One of the authors in the study concluded that if the same metabolic dysfunction were confirmed to occur in humans, there is an urgent need to reduce air pollution, given the growing burden of obesity in today's highly polluted world (Sarah, 2016).

Chinese scientists also found that particulate air pollutants actually changed DNA in mouse mitochondria. The process leading from air pollution to insulin resistance is thought to involve oxidative stress from the free radicals in the pollutants. Mitochondria is the powerhouse of the cell, once its DNA is impaired, then its functions will be impaired (David, 2012). Oxidative stress caused by exposure to arsenic has also been shown to decrease glucosestimulated insulin secretion in vitro. In adipocytes, arsenic increased insulin resistance by suppressing the insulin signaling cascade and glucose uptake (Jean et al., 2015).

Although high levels of POPs have been shown to be associated with diabetes in humans, they have been shown to have positive associations with type 2 diabetes in some animals, in vitro. So thorough animal studies are needed to clarify the pathogenesis and mechanisms by which POPs affect the development of type 2 diabetes and metabolic disorders (Jean et al., 2015).

Conclusion: Several papers reviewed established environmental pollutants may play a role in the pathogenesis of diabetes, however, their mechanisms of actions are not clear. Therefore, more studies are needed to clarify the mechanisms by which pollutants exacerbate diabetes pathogenesis. People are advised to live far away from polluted or industrial environment, and they should make sure their foods and drinks are free from pollutants.

\section{REFERENCES}

American Diabetes Association, ADS (2015). The Cost of Diabetes.

http://www.diabetes.org/advocacy/newsevents/cost-of- diabetes.html?

Andersen, ZJI; Raaschou-Nielsen, O; Ketzel, M; Jensen, SS; Hvidberg, M; Loft, S; Tjonneland, A; Overvad, K; Sørensen, M (2012). Diabetes incidence and long-term exposure to air pollution: a cohort study. Diabetes Care. 35(1):92-8.

Beyerlein, A1; Krasmann, M; Thiering, E; Kusian, D; Markevych, I; D'Orlando, O; Warncke, K; Jochner, S; Heinrich, J; Ziegler, AG (2015). Ambient air pollution and early manifestation of type 1 diabetes. Epidemiology, 26 (3): 31-2

Chang, JW; Chen, HL; Su, HJ; Liao, PC; Guo ,HR; Lee, CC (2010). Dioxin exposure and insulin resistance in Taiwanese living near a highly contaminated area. Epidemiology, 21(1):5661.

Chang, JW; Chen, HL; Su, HJ; Lee, CC (2016). Abdominal Obesity and Insulin Resistance in People Exposed to Moderate-to-High Levels of Dioxin. PLoS One, 11(1)

David, S (2012). Toxic Chemicals and Diabetes. Chinese scientists found that particulate air pollution actually changed DNA in mouse mitochondria.

http://www.diabetesselfmanagement.com/blog/to xic-chemicals-and-diabetes/

González, R N1; Torres-Avilés, F; Carrasco, P E; Salas, P F; Pérez, B F (2013). Association of the incidence of type 1 diabetes mellitus with 
environmental factors in Chile during the period 2000-2007. Rev Med Chil. 141(5):595-601

Hansen, AB; Ravnskjær, L; Loft, S; Andersen, KK; Bräuner, EV; Baastrup, R; Yao, C1; Ketzel, M; Becker, T; Brandt, J; Hertel, O; Andersen, ZJ (2016). Long-term exposure to fine particulate matter and incidence of diabetes in the Danish Nurse Cohort. Environ Int, 91:243-50.

International Diabetes Federation, IDF (2013) Diabetes Atlas. http://www.idf.org/diabetesatlas

International Diabetes Federation, IDF (2015). Nigeria vs World Prevalence of Diabetes http://www.idf.org/membership/afr/nigeria

Jeon, J Y; Kyoung, HH; Kim, DJ (2015). New risk factors for obesity and diabetes: Environmental chemicals. Journal of Diabetes Investigation, 6(2): 109-111

Joel, M; Norma, K; Ram, T (2003). Diabetes Mellitus and 2, 3, 7, 8-Tetrachlorodibenzo-p-Dioxin Elimination in Veterans of Operation Ranch Hand. Journal of Toxicology and Environmental Health, 66:21-221.

Kern, PA1; Said, S; Jackson, WG; Michalek, JE (2004). Insulin sensitivity following Agent Orange exposure in Vietnam veterans with high blood levels of 2, 3, 7, 8-tetrachlorodibenzo-pdioxin. J Clin Endocrinol Metab, 89(9):4665-72

Lee, DH; Lee, IK; Song, K (2006). A strong-doseresponse relation between serum concentrations of persistent organic pollutants and diabetes. Results from the National Health and
Environmental Survey 1999-2000. Diabetes Care, 29: 1638-1644.

Lockwood, AH (2002). Diabetes and air pollution. Diabetes care, 25: 1487-1488

Lodovici, M; Bigagli, E (2011). Oxidative stress and air pollution exposure. $\mathrm{J}$ Toxicol, doi: $10.1155 / 2011 / 487074$

Lynne, P. (2012). BPA's Obesity And Diabetes Link Strengthened By New Study http://www.huffingtonpost.com/2012/02/14/bpachemical-hormone-obesitydiabetes_n_1276996.html

Portal, M (2006). Persistent Organic Pollutants and the burden of diabetes. Lancet, 368: 558-559.

Sarah, B (2016). Study: Air Pollution Heightens Risk of Obesity and Diabetes. http://time.com/4233241/air-pollution-obesitydiabetes/

United States National Institute of Health, USNIH (2014). Types of Diabetes. https://www.niddk.nih.gov/healthinformation/diabetes/types

Waxman, BO (2016). That Plastic Container You Microwave In Could Be Super-Toxic http://time.com/4229503/plastic-in-microwaveis-it-safe/

Wilbert, C (2008). Arsenic Linked to Diabetes http://www.webmd.com/diabetes/news/2008081 9/arsenic-linked-to-diabetes\#1 